\title{
THE PICARD SEQUENCE OF A FIBRATION
}

\author{
ANDY R. MAGID ${ }^{1}$
}

ABSTRACT. A fibration, in the Zariski topology, of algebraic varieties leads to an exact sequence of cohomology of the relative units functor. Under suitable hypotheses, the exact sequence can be interpreted as a sequence including the Picard groups of the base variety, the total space, and the fibre.

Let $X \rightarrow \mathrm{Y}$ be a fibration with fibre $F$ of algebraic varieties (over an algebraically closed field) which is locally trivial in the Zariski topology on $Y$. If $Y$ and $F$ are smooth and $F$ is rational, Fossum and Iverson [2, Proposition 5, p. 8] constructed an exact sequence relating $\operatorname{Pic}(Y), \operatorname{Pic}(X)$, and $\operatorname{Pic}(F)$. The purpose of the present note is to interpret that sequence in terms of the cohomology, in the Zariski topology, of the relative units sheaf. Besides explaining the Fossum-Iverson sequence, this interpretation helps isolate the hypotheses for the existence of the sequence, which leads to some mild generalizations of the original result.

We fix throughout the following conventions: $k$ denotes an algebraically closed field and all varieties considered are over $k$ and irreducible. Unless otherwise specified, all sheaves are for the Zariski topology, and all sheaf cohomology is in that topology. If $V$ is a variety, $U$ an open subset of $V$ and $\mathcal{F}$ a sheaf on $V$, then $(\mathcal{F}) \mid U$ denotes $\mathcal{F}$ restricted to $U$. If $A$ is an abelian group then $(A)_{V}$ denotes the constant sheaf on $V$ associated to $A$. We recall that the higher cohomology groups of constant sheaves on $V$ are trivial, and, hence by descent, that all locally constant sheaves on $V$ are constant. $G_{m, V}$ denotes the units sheaf on $V$.

Definition 1. Let $V$ be a variety. $U_{k, V}$ denotes the presheaf cokernel of $\left(\left(k^{*}\right)_{V} \rightarrow G_{m, V}\right)$. We refer to $U_{k, V}$ as the sheaf of relative units on $V$.

Lemma 2. Let $V$ be a variety.

(i) $U_{k, V}$ is a sheaf on $V$.

(ii) $H^{i}\left(V, U_{k, V}\right)=H^{i}\left(V, G_{m, V}\right)$ for all $i \geq 1$.

(iii) $\operatorname{Pic}(V)=H^{1}\left(V, U_{k, V}\right)$.

Received by the editors October 25, 1974.

AMS (MOS) subject classifications (1970). Primary 14D99, 14F 10, 14F 20.

Key words and phrases. Sheaf cohomology, Leray spectral sequence, Picard group, smooth variety, rational variety.

IPartially supported by NSF Grant GP-37051. 
Proof. Let $D$ denote the sheaf cokernel of $\left(k^{*}\right)_{V} \rightarrow G_{m, V}$. Let $W$ be an open subset of $V$. Then $\left(\left(k^{*}\right)_{V}\right) \mid W=\left(k^{*}\right)_{W}$ and $\left(G_{m, V}\right) \mid W=G_{m, W}$, and hence there is an exact sequence

$$
1 \rightarrow\left(k^{*}\right)_{W} \rightarrow G_{m, W} \rightarrow(D \mid W) \rightarrow 1
$$

of sheaves on $W$. The long exact cohomology sequence begins

$$
0 \rightarrow\left(k^{*}\right)_{W} \rightarrow G_{m, V}(W) \rightarrow D(W) \rightarrow H^{1}\left(W,\left(k^{*}\right)_{W}\right)=0,
$$

and hence $D(W)=U_{k, V}(W)$. This proves part (i). For part (ii), we use the sequence (.t) for $W=V$ and $D=U_{k, V}$ and the fact that $H^{i}\left(V,\left(k^{*}\right)_{V}\right)=$ $H^{i+1}\left(V,\left(k^{*}\right)_{V}\right)=0$ for all $i \geq 1$. Part (iii) is immediate from (ii) and the fact that $\operatorname{Pic}(V)=H^{1}\left(V, G_{m, V}\right)$.

Definition 3. A morphism $E \rightarrow B$ of varieties is a Zariski fibration with fibre $F$ if there is an open cover $\left\{W_{i}\right\}$ of $B$ such that $E \times{ }_{B} W_{i}$ is isomorphic with $F \times W_{i}$ (over $W_{i}$ ) for each $i$.

Lemma 4. Let $f: E \rightarrow B$ be a Zariski fibration with fibre $F$. Then there is an exact sequence

$$
1 \rightarrow U_{k, B} \rightarrow f_{*} U_{k, E} \rightarrow\left(U_{k, F}^{(F))_{B}} \rightarrow 1\right.
$$

of sheaves on $B$.

Proof. The first map is defined as follows: for an open set $W$ of $B$, for an element $a$ of $U_{k, B}(W)$ and for an element $b$ of $G_{m, B}(W)$ representing $a$, send $a$ to class of $b \circ f$ in $f_{*} U_{k, E}(W)=U_{k, E}\left(f^{-1}(W)\right)$. This is trivially an injection. Let $C$ denote its cokernel. If $\left\{W_{i}\right\}$ is an open cover of $B$ as in Definition 3, then $f_{*} U_{k, E}(W)=U_{k, E}(F \times W)$ for every open set $W$ contained in some $W_{i}$. By Rosenlicht's lemma [2, Lemma 3, p.7], the natural map $U_{k, F}(F) \times U_{k, B}(W) \rightarrow U_{k, E}(F \times W)$ is an isomorphism. It follows that $\left(f_{*} U_{k, E}\right)\left|W_{i}=\left(U_{k, B}\right)\right| W_{i} \times\left(U_{k, F}(F)\right)_{W_{i}}$ and, hence, that $C \mid W_{i}=\left(U_{k, F}(F)\right)_{W_{i}}$. Thus $C$ is locally constant, and it follows that $C=\left(U_{k, F}(F)\right)_{B}$.

Theorem 5. Let $f: E \rightarrow B$ be a Zariski fibration with fibre F. Suppose that, for all sufficiently small open sets $W$ of $B$ the natural map $\operatorname{Pic}(F) \times$ $\operatorname{Pic}(W) \rightarrow \operatorname{Pic}(F \times W)$ is an isomorphism. Then there is an exact sequence

$$
\begin{aligned}
0 \rightarrow U_{k, B}(B) \rightarrow U_{k, E}(E) & \rightarrow U_{k, F}(F) \rightarrow \operatorname{Pic}(B) \rightarrow \operatorname{Pic}(E) \\
& \rightarrow \operatorname{Pic}(F) \rightarrow H^{2}\left(B, G_{m, B}\right) \rightarrow H^{2}\left(E, G_{m, E}\right) .
\end{aligned}
$$

Proof. Consider the Leray spectral sequence $H^{p}\left(B, R^{q} f_{*} U_{k, E}\right) \Longrightarrow$ $H^{n}\left(E, U_{k, E}\right)$. The exact sequence of low degree terms is

$$
\begin{aligned}
0 \rightarrow H^{1}\left(B, f_{*} U_{k, E}\right) \rightarrow H^{1}\left(E, U_{k, E}\right) & \rightarrow H^{0}\left(B, R^{1} f_{*} U_{k, E}\right) \\
& \rightarrow H^{2}\left(B, f_{*} U_{k, E}\right) \rightarrow H^{2}\left(E, U_{k, E}\right) .
\end{aligned}
$$


We consider the terms separately, beginning with the third. $R^{1} f_{*} U_{k, E}$ is the sheaf associated to the presheaf $U \rightarrow H^{1}\left(f^{-1} U, U_{k, E}\right)$. By Lemma $2(i+)$, this is the presheaf $f_{*}$ Pic. We have a map of presheaves on $B$, Pic $\rightarrow f_{*} \mathrm{Pic}$. Let $C$ be the presheaf cokernel. On sufficiently small open sets $W$ of $B$ we have $f_{*} \mathrm{Pic}=\mathrm{Pic} \times(\operatorname{Pic}(F))_{W}$ as presheaves on $W$. The sheaf sequence associated to the exact sequence $\mathrm{Pic} \rightarrow f_{*} \mathrm{Pic} \rightarrow C \rightarrow 0$ then yields an isomorphism of $R^{1} f_{*} U_{k, E}$ and the sheaf associated to $C$, since the sheaf associated to $\mathrm{Pic}$ is 0 . But $C$ is locally constant and locally isomorphic to $(\operatorname{Pic}(F))_{B}$, hence $R^{1} f_{*} U_{k, E}=(\operatorname{Pic}(F))_{B}$, and $H^{0}\left(B, R^{1} f_{*} U_{k_{,} E}\right)=\operatorname{Pic}(F)$. By Lemma 2(ii) and (iii) we have $H^{1}\left(E, U_{k, E}\right)=\operatorname{Pic}(E)$ and $H^{2}\left(E, U_{k, E}\right)=H^{2}\left(E, G_{m, E}\right)$. The long exact sequence of cohomology arising from the sheaf sequence of Lemma 4 shows that $H^{2}\left(B, f_{*} U_{k, E}\right)=H^{2}\left(B, U_{k, B}\right)$ since $H^{1}\left(B,\left(U_{k, F}(F)\right)_{B}\right)=$ $H^{2}\left(B,\left(U_{k, F}(F)\right)_{B}\right)=0$, and hence, by Lemma $2(\mathrm{ii}), H^{2}\left(B, f_{*} U_{k, E}\right)=$ $H^{2}\left(B, G_{m, B}\right)$. The first part of that long exact sequence is

$$
0 \rightarrow U_{k, B}(B) \rightarrow U_{k, E}(E) \rightarrow U_{k, F}(F) \rightarrow H^{1}\left(B, U_{k, B}\right) \rightarrow H^{1}\left(B, f_{*} U_{k, E}\right) \rightarrow 0 .
$$

By Lemma 2(iii), $H^{1}\left(B, U_{k, B}\right)=\operatorname{Pic}(B)$, and now splicing this sequence to the sequence obtained from the Leray spectral sequence yields the result.

Remark. Let $f: E \rightarrow B$ be a Zariski fibration with fibre $F$. Suppose that for all sufficiently small open sets $W$ of $B$ the natural map $\operatorname{Pic}(F) \times$ $\mathrm{Pic}(W) \rightarrow \mathrm{Pic}(F \times W)$ is an isomorphism, and that $F$ and $B$ are smooth. Then the map $\operatorname{Pic}(E) \rightarrow \operatorname{Pic}(F)$ of Theorem 5 is onto.

Proof. We need to show that the kernel of $H^{2}\left(B, G_{m}\right) \rightarrow H^{2}\left(E, G_{m}\right)$ is zero. Let $W$ be an open set of $B$ with $f^{-1}(W)=F \times W$. We have a commutative diagram

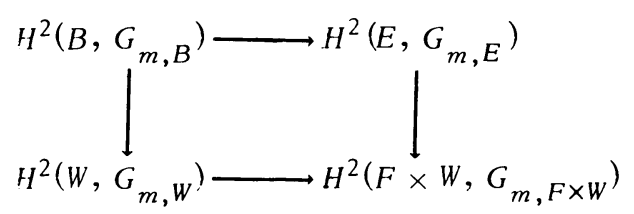

and the bottom map is clearly injective. By $[1,1.8, \mathrm{p} .104]$, the vertical maps are injective and the result follows.

The exact sequence of Theorem 5 is the generalization of the FossumIverson sequence, and the above remark explains why their sequence stops with $\operatorname{Pic}(F)$.

Fossum and Iverson show that if $F$ and $W$ are smooth varieties, and one is rational, then $\operatorname{Pic}(F \times W)=\operatorname{Pic}(F) \times \operatorname{Pic}(W)$ [2, Corollary 6, p. 11]. This then gives some conditions for the hypotheses of Theorem 5 to obtain. (We remark that this product formula does not follow from the spectral sequence argument given here, but is a hypothesis for it.) For example, one then obtains the Fossum-Iverson sequence under the assumptions that $B$ is rational and $F$ smooth. 


\section{REFERENCES}

1. M. Artin, Grothendieck topologies, Mathematics Department Lecture Notes, Harvard Univ., Cambridge, Mass., 1962.

2. R. Fossum and B. Iverson, On Picard groups of algebraic fibre spaces, Aarhus Universitet Matematisk Institut Preprint Series, no. 19, Aarhus, Denmark, $1972 / 73$.

DEPARTMENT OF MATHEMATICS, UNIVERSITY OF OKLAHOMA, NORMAN, OKLAHOM A 73069

Current address: Department of Mathematics, University of Illinois, Urbana, Illinois 61801 